\title{
The effect of dielectric permittivity on the fields radiated from a radio-frequency electric dipole in a homogeneous whole space
}

\author{
Tomas Naprstek ${ }^{1}$ and Richard S. Smith ${ }^{1}$
}

\begin{abstract}
The radio imaging method (RIM) is an electromagnetic crossborehole method with applications in mineral exploration, as well as in the coal industry, where it is used across mine drives. Attenuation of the signal from conductive regions may indicate areas of mineralization, and these conductive effects in general dominate the response. In an effort to better understand the effect of a material's dielectric permittivity on the response of the RIM, we have developed a simple program to model an electric dipole in a homogeneous whole space. When increasing the dielectric permittivity, the amplitude peak broadened and increased, whereas the phase peak sharpened and shifted negatively. To showcase the effect of dielectric permittivity on RIM data, data
\end{abstract}

recorded from two transmitter positions in a moderately homogeneous zone in the Sudbury Basin were curve fit, and we concluded that despite the stronger effect that conductivity has on the signal, RIM is still sensitive to dielectric permittivity, and appropriate values must be used when developing conductivity tomograms. In addition, we found that for the given situation and frequencies used, an increase in either the conductivity or dielectric permittivity could be accounted for by a decrease of approximately the same factor in the other variable. However, the low-conductivity, high-permittivity case seemed to fit the shape of the amplitude and phase curves better. For the sulfide impregnated crystalline rocks at our field site, relative dielectric constants of 26.4 and 31 at 1250 and $625 \mathrm{kHz}$, respectively, were inferred.

\section{INTRODUCTION}

The radio imaging method (RIM) is a tomographic geophysical method that can be used to map electromagnetic (EM) properties in areas of interest between boreholes or mine drifts. Developed for the coal mining industry (Stolarczack and Fry, 1986), RIM has since been applied with some success in several other areas, including mineral exploration and delineation (Thomson et al., 1992; Fullagar et al., 2000) and characterization of rock quality (Korpisalo and Heikkinen, 2014). RIM is effective at locating areas of high conductivity, which can be indicative of sulfide-bearing zones. The conductive medium causes the EM radiation emitted by the transmitter to attenuate, whereas resistive zones allow the radiation to propagate without attenuation. In base metal mining, zones of strong attenuation may indicate mineralization (Zhou et al., 1998; Stevens et al., 2000).

RIM surveys are conducted by operating a radio-frequency antenna at several set locations down a borehole or along a mine drift (Figure 1). The radio signal is received by an antenna at multiple locations in another borehole or drift. The variation in signal amplitude and phase can be used to determine features of the material between the two antennas. In the mining industry, the results can be used to define conductive drill targets, and if there is confidence in the RIM results, further drilling could be minimized or avoided (Mutton, 2000).

For field data, an absorption tomogram is built from the data (Thomson and Hinde, 1993; Fullagar et al., 2000), which can be used to delineate ore bodies. Alternatively, modeling can be applied (Smith et al., 1990; Vogt and Marvin, 2006; Dorn and Ascher, 2007) to further interpret the data, developing a conductivity tomogram or an EM model of the material.

The goal of this research was to investigate the impact that dielectric permittivity has on the radio signal measured at the receivers. To accomplish this, a whole-space model was developed. This type of model was chosen because it is simple to develop for our specific purpose, though it does come with many restrictions due to it being homogeneous. In particular, a homogeneous model is only

First presented at the SEG 84th Annual International Meeting. Manuscript received by the Editor 9 April 2015; revised manuscript received 30 November 2015; published online 18 February 2016.

Laurentian University, Sudbury, Ontario, Canada. E-mail: tnaprstek@laurentian.ca; rssmith@laurentian.ca.

(C) 2016 Society of Exploration Geophysicists. All rights reserved. 
valid for RIM data recorded at depths greater than the maximum transmitter-receiver separation to avoid surface effects (Korpisalo, 2005). Nonetheless, subject to those restrictions, this model can be used to estimate the effect of the dielectric constant on RIM data. More rigorous studies of dielectric effects could be undertaken using finite-element simulations such as COMSOL Multiphysics (Li and Smith, 2015) or with the method described by Dorn et al. (2008).

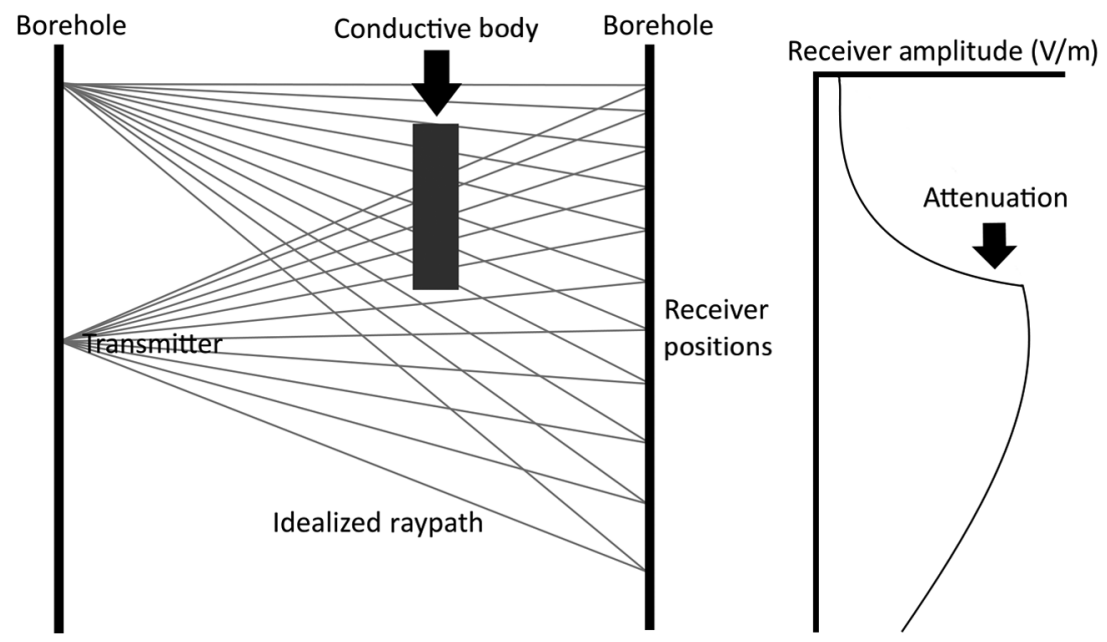

Figure 1. The basic setup of a crosshole RIM survey. The transmitter is operated at several locations in one hole, and measurements are taken with the receiver at multiple locations in another hole. An amplitude-versus-depth plot is shown on the right for the transmitter at the lower position. Conductive zones/bodies strongly attenuate the signal.

\section{DEVELOPING A MODEL}

To investigate the RIM signal, we developed a whole-space modeling program in MATLAB using the formulation of Ward and Hohmann (1987). The program simulates the transmitter as an electric dipole (RIM systems may also use a magnetic dipole) in a homogeneous whole space and calculates the resulting electric field that would be measured in a receiver borehole, as a function of the parameters of the whole space and the system geometry (Figure 2). By varying the parameters of the model, it is possible to gain an understanding of how the signal changes, which can ultimately assist in creating more accurate inversions and interpretations.

The mathematical formula describing the electric field $E$ of a $z$-directed electric dipole in a whole space is (adapted from Ward and Hohmann [1987], p. 173; and see the additional description from Zhou and Fullagar, 2001) as follows:

$$
\begin{aligned}
E & =\frac{I d s}{4 \pi(\sigma+i \omega \varepsilon) r^{3}} e^{-i k r}\left[\left(\frac{z^{2}}{r^{2}} \mathbf{u}_{\mathbf{z}}+\frac{z y}{r^{2}} \mathbf{u}_{\mathbf{y}}\right.\right. \\
& \left.+\frac{z x}{r^{2}} \mathbf{u}_{\mathbf{x}}\right)\left(-k^{2} r^{2}+3 i k r+3\right) \\
& \left.+\left(k^{2} r^{2}-i k r-1\right) \mathbf{u}_{\mathbf{z}}\right]
\end{aligned}
$$

where

$$
r=\sqrt{x^{2}+y^{2}+z^{2}},
$$

$$
k=\alpha-i \beta,
$$

is the complex wavenumber,

$$
\alpha=\omega\left\{\frac{\mu \varepsilon}{2}\left[\left(1+\frac{\sigma^{2}}{\varepsilon^{2} \omega^{2}}\right)^{1 / 2}+1\right]\right\}^{1 / 2}
$$

is the wavenumber/phase coefficient, and

$$
\beta=\omega\left\{\frac{\mu \varepsilon}{2}\left[\left(1+\frac{\sigma^{2}}{\varepsilon^{2} \omega^{2}}\right)^{1 / 2}-1\right]\right\}^{1 / 2}
$$

is the attenuation rate/absorption coefficient.

Here, $I$ is the current (A), $d s$ is the infinitesimal dipole length (m), $\sigma$ is the conductivity $(\mathrm{S} / \mathrm{m}), \omega$ is the angular frequency (radians/s) dependent on the transmitter frequency $(\mathrm{Hz}), \varepsilon$ is the dielectric permittivity $(\mathrm{F} / \mathrm{m}), \mu$ is the magnetic permeability $(\mathrm{H} / \mathrm{m})$, and $\mathbf{u}_{i}$ is the unit vector in the $i$ th direction. Following a short study (Naprstek, 2014) on the effect of integrating over infinitesimal dipoles for the transmitter, the transmitter was split up into $1 \mathrm{~m}$ dipole segments and their results summed to account for geometric effects.

Equation 1 was adapted from the $x$-directed dipole given by Ward and Hohmann by switching variables and ensuring a right-handcoordinate system. For the purposes of this modeling program, the boreholes are always in the same $y$-plane, and therefore there is no
Figure 2. The RIM geometric configuration, defining all variables involved in the calculations. 
$y$-distance. In addition, the $x$-distance is measured as the horizontal separation between the boreholes at the top, and therefore the holes are assumed to start at the same depth/elevation.

The "measured" amplitude and phase were calculated from the real and imaginary parts of the $z$ - and $x$-components of the electric field computed at $1 \mathrm{~m}$ intervals down the receiver borehole. Two reference frames are required for these calculations: the global reference frame and the transmitter reference frame. The global reference frame measures $z$ vertically (positive straight down), whereas the transmitter reference frame measures $z$ along the angle of the transmitter borehole (positive down the borehole).

The component of the electric field parallel (axial) to the receiver borehole is then calculated as follows:

$$
\begin{aligned}
& E_{\text {parallel }}=E_{z} \cos \left(\theta_{\text {trans }}+\theta_{\text {receive }}\right) \\
& \quad-E_{x} \cos \left(90^{\circ}-\left(\theta_{\text {trans }}+\theta_{\text {receive }}\right)\right)
\end{aligned}
$$

where $\theta_{\text {trans }}$ is the transmitter borehole angle, $\theta_{\text {receive }}$ is the receiver borehole angle, $E_{z}$ is the $z$ component of the electric field (in the transmitter reference frame, parallel to the transmitter) at the receiver position being calculated, and $E_{x}$ is the $x$ component at the same location (and therefore orthogonal to the transmitter).

The real and imaginary axial components are computed, and finally the total amplitude and phase angle are calculated and plotted (Figure 3). The phase angle is defined as the $\tan ^{-1}$ function of the imaginary component over the real component in radians, and it is measured from $-\pi$ to $+\pi$. The depth is defined as a distance away from the transmitter location, such that a depth of $0 \mathrm{~m}$ is at the same vertical location as the transmitter.

\section{DIELECTRIC PERMITTIVITY STUDY}

The following plots (Figures 4-6) show the effect of changing the dielectric permittivity on the amplitude and phase as varied over a large range of values, followed by a smaller range, such that the direction of the phase variation can be identified. The values for the other parameters used in the simulation are listed in Table 1 . The applicability of these results depends on the loss tangent as follows (Ward and Hohmann, 1987):

$$
\delta=\frac{\sigma}{\omega \varepsilon},
$$

Which, given our standard parameter values, gives us a loss tangent of $\delta \approx 1.44 / \varepsilon_{r}$. Therefore, the loss tangent will be close to unity, placing it in a transition zone, in which the conduction and displacement currents are of importance (Keller, 1987). This results in $\alpha$ and $\beta$ being sensitive to changes in the relative dielectric permittivity.

From Figures 4-6, several patterns can be observed in the way the amplitude and phase values change when varying the dielectric permittivity. The following was noted:

1) Increasing the dielectric permittivity causes a broadening of the amplitude peak and increases the maximum amplitude (Figure 4), and

2) increasing the dielectric permittivity causes a sharpening of the phase peak (Figure 5) and shifts phase negatively (Figure 6).

These effects (sharpening, increasing, etc.) of increasing the dielectric permittivity are similar for phase, and they are opposite for amplitude when increasing conductivity or magnetic permeability (Naprstek, 2014).

\section{CASE STUDY: SURVEY FROM DRURY, SUDBURY}

As an example of the effect of dielectric permittivity on RIM data, we curve fit the field data by varying the conductivity and dielectric permittivity. The data were collected by GEOFARA using the Fara RIM system on behalf of Sudbury Integrated Nickel Operations, a Glencore Company, in Drury Township, near Sudbury, Ontario, Canada.

Figure 7 shows a resistivity tomogram created by GEOFARA. It was developed using the FARA software as described by Redko et al. (2000), and following the interpretation methods described by Korpisalo (2005). This plot shows the resistivity between boreholes DR-157 and DR-159 for transmitter frequency of $1250 \mathrm{kHz}$. The $y$-axis is the depth below the surface.
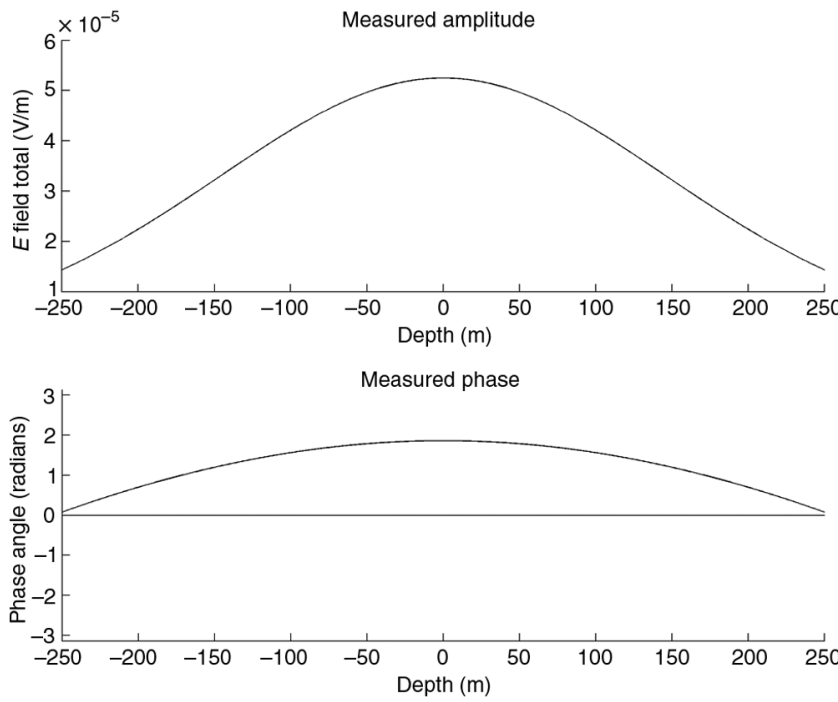

Figure 3. Sample of the output from the modeling code showing amplitude and phase of the electric field that would be measured at receiver positions ranging from $250 \mathrm{~m}$ above $(-250 \mathrm{~m})$ to $250 \mathrm{~m}$ below $(+250 \mathrm{~m})$ the depth of the transmitter. This is for a parallel borehole system $\left(\theta_{\text {trans }}=\theta_{\text {receive }}=0\right)$, borehole separation $x=500 \mathrm{~m}$, conductivity $=10^{-4} \mathrm{~S} / \mathrm{m}$, and frequency $=1250 \mathrm{kHz}$.

Table 1. Standard parameter values used in calculations.

Variable

Value

$x$ distance between holes

$500 \mathrm{~m}$

Transmitter length

$10 \mathrm{~m}$

Frequency

$1250 \mathrm{kHz}$

Transmitter current

10 amps

Transmitter borehole angle

$0^{\circ}$

Receiver borehole angle

$0^{\circ}$

Conductivity

$10^{-4} \mathrm{~S} / \mathrm{m}$

Relative magnetic permeability 
The area is divided by material of higher resistivity (lower conductivity) above approximately $-325 \mathrm{~m}$ and the material of lower resistivity (higher conductivity) below $-325 \mathrm{~m}$, with the greatest conductivity concentrated approximately $-450 \mathrm{~m}$, closer to DR-151.

To model the data, the geometric parameters were adjusted to represent the field situation as closely as possible. Due to the limitations of this model, the boreholes had to be set to a constant angle. The boreholes were set $469 \mathrm{~m}$ apart (the separation indicated by the tomogram at depth $-150 \mathrm{~m}$ ), and at angles $24.5^{\circ}$ from vertical, and $-9.9^{\circ}$ from vertical, for DR-151 and DR-160, respectively.
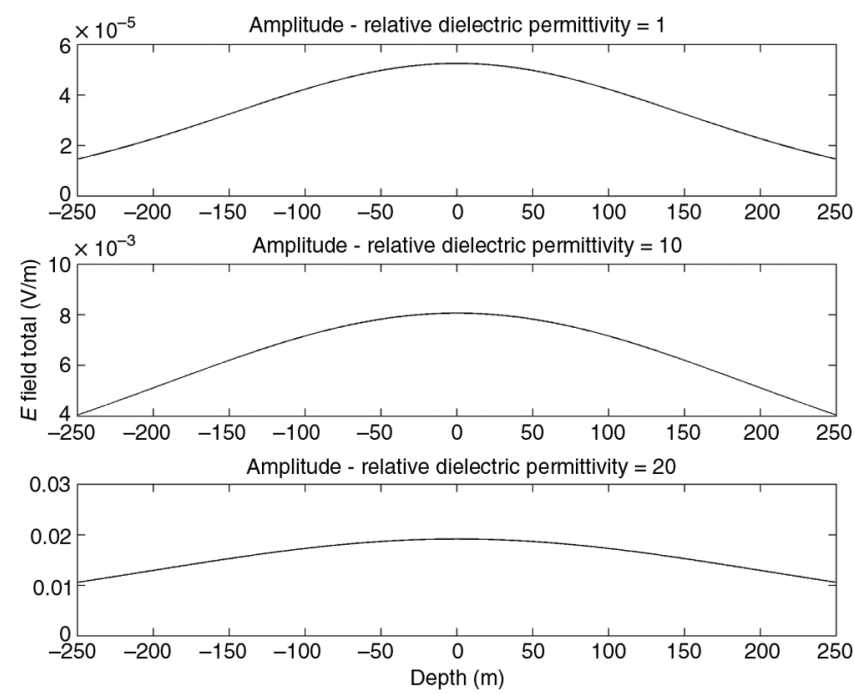

Figure 4. Changes in amplitude of the axial electric field as a function of receiver depth for three different relative dielectric permittivity values. The amplitude can be seen to increase significantly as the relative dielectric permittivity increases.

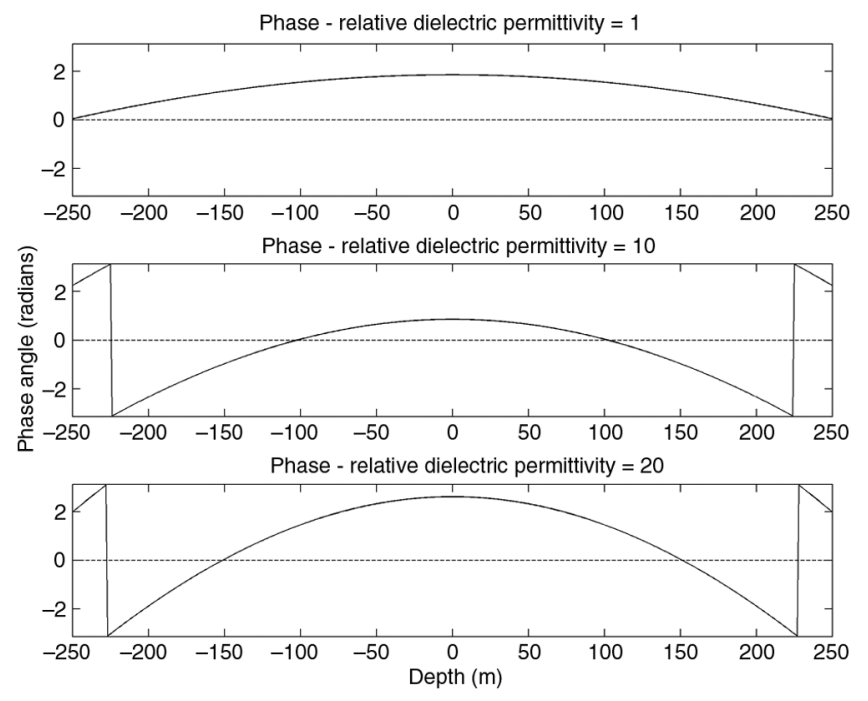

Figure 5. Changes in phase of the axial electric field as a function of receiver depth for three different relative dielectric permittivity values. The shape of the phase peak is sharpening as the relative dielectric permittivity increases.
Two transmitter gathers were curve fit: $1250 \mathrm{kHz}$ for the transmitter located at $-535 \mathrm{~m}$ in borehole DR-151 and $625 \mathrm{kHz}$ at $-530 \mathrm{~m}$ in DR-160. These were chosen for the case study because they are positioned in the moderately homogeneous lower zone, which would lead to cleaner data. Though the modeling program would have a better ability of curve fitting the upper resistive zone that is more homogeneous, it would be too close to the surface, and it would require the ability to take into account surface reflections. As such, the curve fitting was done by primarily using the receiver data between -500 and $-600 \mathrm{~m}$ (though other depths are shown to see the effect of the conductive zones on the field data). When simulating the field measurements, the model data and the field data appeared to be shifted with respect to each other in the borehole depth. This was assumed to be due to incorrect geometry because the boreholes were neither coplanar (in the same $y$-plane) nor were they drilled perfectly straight. To account for this shift, all calculated data have been shifted up the receiver hole by $50 \mathrm{~m}$.

Two curve fitting procedures were completed for the two sets of data: (1) setting the relative dielectric permittivity to 6.5 and varying the conductivity and (2) setting the conductivity to an approximate value given by the tomogram, $8 \times 10^{-4} \mathrm{~S} / \mathrm{m}$, and varying the dielectric permittivity. When setting the relative dielectric permittivity to a reasonable value for the area of 6.5 (Fullagar and Livelybrooks, 1994), the first data set's conductivity had to be set approximately 4.2 times greater than expected from the GEOFARA tomograms: $3.36 \times 10^{-3} \mathrm{~S} / \mathrm{m}$ (Figure 8). This caused the amplitude of the model to be too small in magnitude. When setting the conductivity in the range expected from the tomograms $\left(8 \times 10^{-4} \mathrm{~S} / \mathrm{m}\right)$, the data were found to fit well at a relative dielectric permittivity of 26.4 (Figure 9), or approximately 4.1 times greater than the estimated value for the area. When this same fitting was completed for the second set of data (Figures 10 and 11), the case with the relative permittivity fixed at 6.5 required that the conductivity be adjusted to $4.3 \times 10^{-3} \mathrm{~S} / \mathrm{m}$, approximately 5.4 times greater than expected.

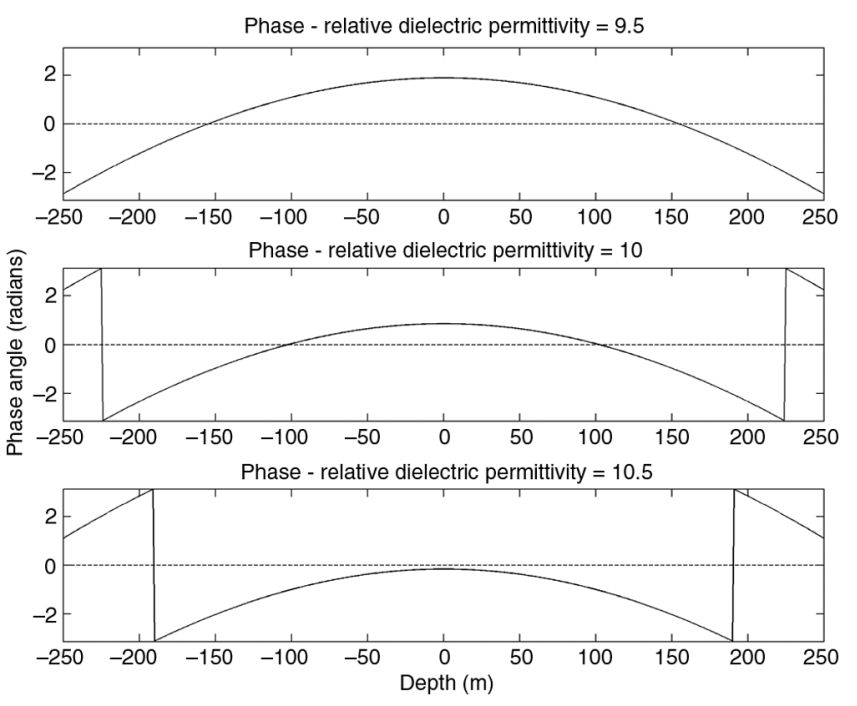

Figure 6. Changes in phase of the axial electric field as a function of receiver depth for three slightly different relative dielectric permittivity values. The phase values are shifted in the negative direction by relatively large amounts for small increases in the relative dielectric permittivity. 
With the conductivity set to the expected approximate value of $8 \times 10^{-4} \mathrm{~S} / \mathrm{m}$, the dielectric permittivity was adjusted to 31 , approximately 4.8 times greater than the expected value.

From these two examples, it can be seen that there appears to be a close connection between the change in conductivity and the dielectric permittivity. At these frequencies and in this situation, a decrease in one can be accommodated by increasing the other by roughly the same factor, and the resulting phase plot will be approximately the same. However, for the amplitude curves, the fixed conductivity and adjusted permittivity models (Figures 9 and 11)
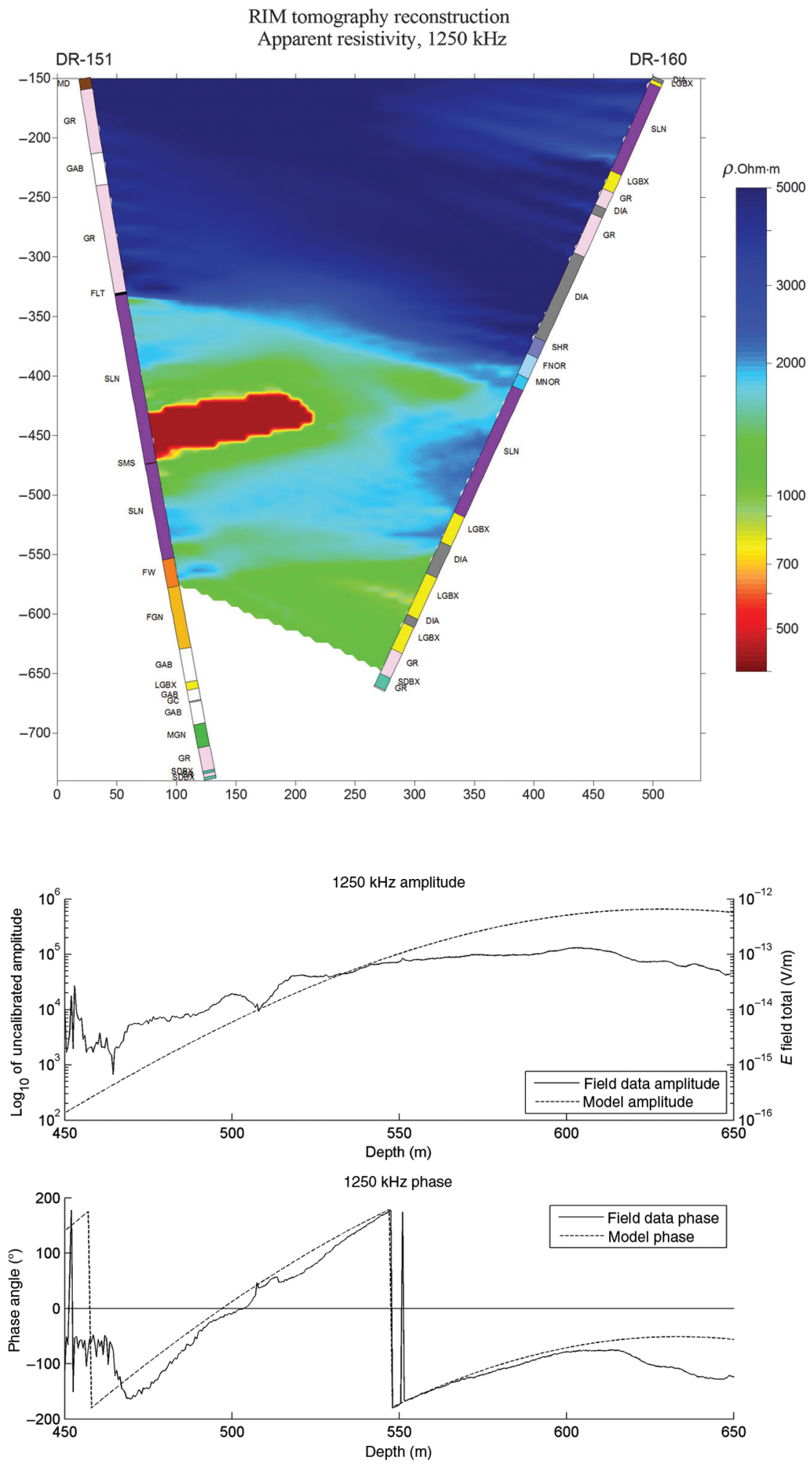

Figure 7. The $1250 \mathrm{kHz}$ resistivity tomogram.

Figure 8. Model and field data comparisons, with the transmitter at $535 \mathrm{~m}$ depth in borehole DR151 , frequency $=1250 \mathrm{kHz}$. The model conductivity is set to $3.36 \times 10^{-3} \mathrm{~S} / \mathrm{m}$, and the relative dielectric permittivity is set to 6.5 . 
were more similar to the field data than the models with the fixed permittivity and adjusted conductivity (Figures 8 and 10). Changing the conductivity changes the amplitude significantly. Unfortunately, the amplitudes for the field data are not calibrated to $\mathrm{V} / \mathrm{m}$, and so the absolute amplitude information cannot be used to resolve the conductivity/relative permittivity ambiguity. Instead, we scaled the model data to be similar to the field data, and we selected the model that best fit the phase data and the amplitude shape.

It would have been greatly beneficial if the core was wireline logged for physical properties, as comparisons could be made and used to constrain this modeling. However, by using the geology logging for the boreholes, estimates for appropriate dielectric per- mittivity values can still be found in the literature. From Figure 7, it can be seen from the boreholes that the area for the data is dominated by SLN (norite), with some LGBX (late granite breccia) and DIA (diabase). According to Telford et al. (1990), at frequencies greater than $100 \mathrm{kHz}$, granite can range in dielectric constants from 4.8 to 18.9 , and diabase can range from 10.5 to 34.5 . According to Parkhomenko (1967), norite can range from 10.9 to 12 , although no frequency range for this measurement is given. It was also noted in the core logging that the SLN contained sulphides, which could lead to above-average dielectric permittivity values. As such, the dielectric values inferred, though large, could be explained due to the diabase and effects of the sulphides.
Figure 9. Model and field data comparisons, with the transmitter at $535 \mathrm{~m}$ depth in borehole DR151 , frequency $=1250 \mathrm{kHz}$. The model conductivity is set to $8 \times 10^{-4} \mathrm{~S} / \mathrm{m}$, and the relative dielectric permittivity is set to 26.4 .

Figure 10. Model and field data comparisons, with the transmitter at $530 \mathrm{~m}$ depth in borehole DR-160, frequency $=625 \mathrm{kHz}$. The model conductivity is set to $4.3 \times 10^{-3} \mathrm{~S} / \mathrm{m}$, and the relative dielectric permittivity is set to 6.5 .
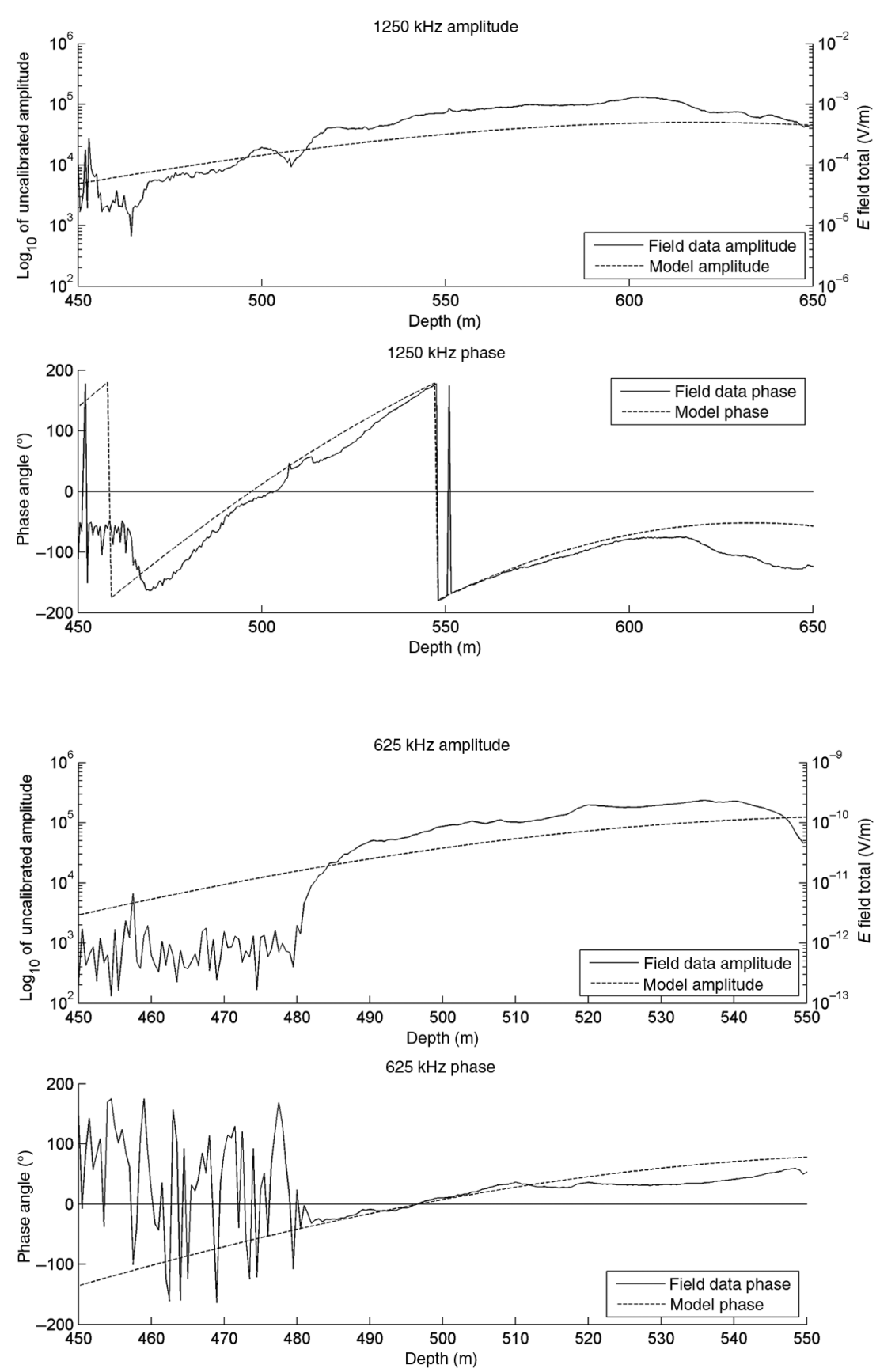

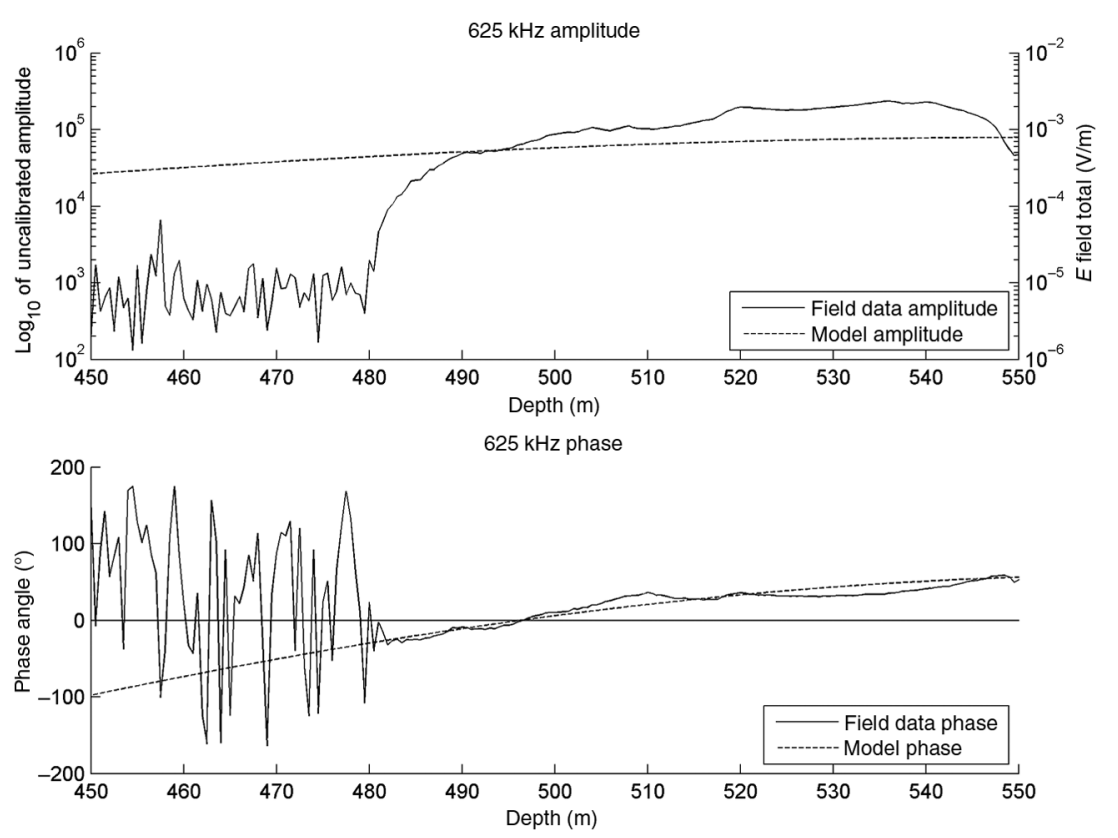

Figure 11. Model and field data comparisons, with the transmitter at $530 \mathrm{~m}$ depth in borehole DR-160, frequency $=625 \mathrm{kHz}$. The model conductivity is set to $8 \times 10^{-4} \mathrm{~S} / \mathrm{m}$, and the relative dielectric permittivity is set to 31 .

\section{CONCLUSIONS}

In this paper, we have reviewed the RIM as a tool for mineral exploration and examined the effect of a material's dielectric permittivity on the electric field that would be measured by a RIM survey. By using the formula for the electric field of a dipole in a whole space, we simulated the response as measured by RIM, provided that the antennas are at depths greater than their horizontal separation.

We varied the dielectric permittivity to observe how the amplitude and phase of the RIM signal will vary. Increasing the dielectric permittivity causes the amplitude peak to broaden, the phase peak to sharpen, and increases the amplitude values, but shifts phase values negatively.

We found that despite conductivity's dominance of the signal, RIM is still sensitive to changes in dielectric permittivity, and that in situations similar to the case study, increasing either variable can be accounted for by decreasing the other by approximately the same factor. In the two examples we looked at, the set with lower conductivity and higher relative dielectric permittivity seemed to better fit the shapes of the amplitude and phase curves. Therefore, when inverting RIM data for conductivity, appropriate dielectric permittivity values must be used.

Investigating these effects in more comprehensive modelling programs may yield more robust results that could prove useful in RIM field data interpretation.

\section{ACKNOWLEDGMENTS}

We would like to acknowledge KGHM International, Sudbury Integrated Nickel Operations A Glencore Company, Vale Limited, Wallbridge Mining Company, the Centre for Excellence in Mining
Innovation (CEMI), the Mineral Exploration Research Centre (MERC), and NSERC for support and funding. We would also like to thank Sudbury Integrated Nickel Operations for releasing the field data used in our case study. We would finally like the thank P. K. Fullagar and three anonymous reviewers for their help in significantly revising and focusing this paper.

\section{REFERENCES}

Dorn, O., and U. Ascher, 2007, Shape reconstruction in $3 \mathrm{D}$ electromagnetic induction tomography using a level set technique: Proceedings of 23rd International Review of Progress in Applied Computational Electromagnetics, ACES, Extended Abstracts, 695-700.

Dorn, O., H. Bertete-Aguirre, and G. C. Papanicolaou, 2008, Adjoint fields and sensitivities for 3D electromagnetic imaging in isotropic and anisotropic media,

in L. L. Bonilla, ed., Inverse problems and imaging: Springer Berlin Heidelberg, 35-65.

Fullagar, P. K., and D. Livelybrooks, 1994, Trial of tunnel radar for cavity and ore detection in the Sudbury mining camp, Ontario: Proceedings of the 5th International Conference on Ground Penetrating Radar (GPR'94), Waterloo Centre for Groundwater Research 3, 883-894.

Fullagar, P., D. Livelybrooks, P. Zhang, A. Clavert, and Y. Wu, 2000, Radio tomography and borehole radar delineation of the McConnell nickel sulfide deposit, Sudbury, Ontario, Canada: Geophysics, 65, 19201930, doi: $10.1190 / 1.1444876$.

Keller, G. V., 1987, Rock and mineral properties, in M. N. Nabighian, ed., Electromagnetic methods in applied geophysics I: SEG, 12-51.

Korpisalo, A., 2005, Radio imaging method with FARA equipment: Crosshole surveys in borehole sections $\mathrm{Kr} 4-\mathrm{Kr} 10$ and $\mathrm{Kr} 10-\mathrm{Kr} 2$ in Olkiluoto with FARA in 2005: Geological Survey of Finland, Report Q16.1/ 2005/1.

Korpisalo, A., and E. Heikkinen, 2014, Radiowave imaging research (RIM) for determining the electrical conductivity of the rock in borehole section OL-KR4-OL-KR10 at Olkiluoto, Finland: Exploration Geophysics, 46, 141-152, doi: 10.1071/EG13057.

Li, Y., and R. S. Smith, 2015, Forward modelling of radio imaging (RIM) data with the Comsol RF module: Computers and Geosciences, 85, 6067, doi: 10.1016/j.cageo.2015.08.012.

Mutton, A. J., 2000, The application of geophysics during evaluation of the Century zinc deposit: Geophysics, 65, 1946-1960, doi: 10.1190/1 .1444878 .

Naprstek, T., 2014, Modelling Radio Imaging Method data using electric dipoles in a homogeneous whole space: M.S. thesis, Laurentian University.

Parkhomenko, E., 1967, Electrical properties of rocks: Plenum Press.

Redko, G., K. Ratnikov, A. Savitsky, A. Fedorov, A. Shuval-Sergeev, K. Stevens, and A. Watts, 2000, Method of cross-hole radiowave surveying in ore deposits: 300 years of Russian mine-geological service conference, http://www.farasystem.ru/Pubs/Spb2000_Text_E.pdf, accessed 25 November 2015.

Smith, G. H., P. R. Williamson, and K. Vozoff, 1990, The application of nested dissection to the solution of a 2.5D electromagnetic problem: Applied Computational Electromagnetics, 5, 87-106.

Stevens, K., A. Watts, and G. Redko, 2000, In-mine applications of the radio-wave method in the Sudbury igneous complex: 70th Annual International Meeting, SEG, Expanded Abstracts, 1130-1133.

Stolarczack, L. G., and R. C. Fry, 1986, Radio imaging method (RIM) or diagnostic imaging of anomalous geologic structures in coal seam waveguides: Transactions of the Society for Mining, Metallurgy, and Exploration, Inc., 288, 1806-1814.

Telford, W. M., L. P. Geldart, and R. E. Sheriff, 1990, Applied geophysics: Cambridge University Press.

Thomson, S., and S. Hinde, 1993, Bringing geophysics into the mine: radio attenuation imaging and mine geology: Exploration Geophysics, 24, 805810, doi: 10.1071/EG993805.

Thomson, S., J. Young, and N. Sheard, 1992, Base metal applications of the radio imaging method, Current status and case studies: Exploration Geophysics, 23, 367-372, doi: 10.1071/EG992367. 
Vogt, D., and A. Marvin, 2006, Characterising borehole radio antenna performance using finite-difference time-domain modelling: South African Journal of Geology, 109, 561-570, doi: 10.2113/gssajg.109.4.561.

Ward, S. H., and G.W. Hohmann, 1987, Electromagnetic theory for geophysical applications, in M. N. Nabighian, ed., Electromagnetic methods in applied geophysics I: SEG, 131-312.
Zhou, B., and P. K. Fullagar, 2001, Delineation of sulphide ore-zones by borehole radar tomography at Hellyer Mine, Australia: Journal of Applied Geophysics, 47, 261-269, doi: 10.1016/S0926-9851(01)00070-2.

Zhou, B., P. K. Fullagar, and G. N. Fallon, 1998, Radio frequency tomography trial at Mt Isa Mine: Exploration Geophysics, 29, 675-679, doi: 10 $.1071 /$ EG998675. 